\title{
Music Therapy in Adults With COPD
}

\author{
Jing Huang, Xiaohui Yuan, Nan Zhang, Hui Qiu, and Xiangdong Chen
}

\begin{abstract}
BACKGROUND: Music therapy, as a non-drug therapy, is widely used in patients with COPD. However, the effects of music therapy on dyspnea, anxiety, depression and other physiological parameters has not been elucidated. Therefore, we sought to investigate the effects of passive music therapy (listening to music) and mixed music (combination of listening and singing) in adults with COPD. METHODS: A systematic literature search was performed in PubMed, Cochrane, Embase, Web of Science, China National Knowledge Infrastructure, VIP, and Wanfang Data up to June 26, 2019. All randomized controlled trials that compared music with usual care or other non-musical types of intervention in subjects with COPD were included in the study. The risk of bias for randomized controlled trials was assessed with the Cochrane risk of bias tool. Outcomes included dyspnea, anxiety, depression, sleep quality, and quality of life, as well as physiological parameters (eg, blood pressure and breathing frequency). RESULTS: After initial screening of 216 potentially relevant records, 59 studies were eligible, and 12 studies with $\mathbf{8 1 2}$ participates met the inclusion criteria and were included in the final meta-analysis. Analysis showed that music therapy had a significant effect in relieving dyspnea (mean difference: $-0.69,95 \% \mathrm{CI}-0.80$ to $-0.58, P<.001$ ) and anxiety (standardized mean difference: $-1.87,95 \%$ CI -2.72 to $-1.02, P<.001$ ) in adults with COPD. Compared with the control group, music had no statistically significant effect on depression or St George Respiratory Questionnaire score. However, when it came to improving sleep quality, music reduced the total Pittsburgh Sleep Quality Index score $(P<.001)$. In addition, the pooled results showed that there was a significant improvement in systolic blood pressure (mean difference: $\mathbf{- 7 . 4 5 , 9 5 \%} \mathbf{C I}-\mathbf{1 0 . 9 5}$ to $-3.96, P<.001$ ) and diastolic blood pressure (mean difference: $-4.07,95 \%$ CI -7.03 to -1.12 , $P=.007)$ in the music group compared to the control. CONCLUSIONS: Music therapy is effective in reducing dyspnea and anxiety in subjects with COPD. Additionally, music therapy may also improve sleep quality and physiological parameters of subjects with COPD. However, our conclusions need to be supported further by larger and longer well-designed trials. Key words: music therapy; COPD; dyspnea; anxiety; sleep quality; quality of life; blood pressure; systematic review. [Respir Care 2021;66(3):501-509. (C) 2021 Daedalus Enterprises]
\end{abstract}

\section{Introduction}

COPD is one of the most common chronic diseases in the world, with high prevalence and related disability and mortality. It is projected to be the third leading cause of death

Ms Huang and Mr Qiu are affiliated with the School of Medicine, Shenzhen University, Shenzhen, China. Ms Huang and Mr Qiu are affiliated with the Department of General Medicine, Shenzhen University General Hospital, Shenzhen, China. Ms Yuan is affiliated with the Department of General Medicine, Huizhou Municipal Central Hospital, Huizhou, China. Mr Zhang is affiliated with the Nanyuan Community Health Center, Shenzhen Sixth People's Hospital, Shenzhen, China. Dr Chen is affiliated with the Otolaryngology-Head and Neck Surgery, Shenzhen University General Hospital, Shenzhen, China. worldwide by $20300^{1,2}$ In China, the overall prevalence of COPD as defined by spirometry in adults age $20 \mathrm{y}$ or older was $8.6 \%$ in 2015 , or 99.9 million people. Compared with a study conducted 10 years ago, the prevalence of people with COPD in China has increased by $67 \%{ }^{3}$

\footnotetext{
The authors have disclosed no conflicts of interest.

Supplementary material related to this paper is available at http://www. rcjournal.com.

Correspondence: Xiangdong Chen MD, Shenzhen University General Hospital, No. 1098 Xueyuan Ave, Xili University Town, Nanshan District, Shenzhen, 518055, China. E-mail: xdc1981@szu.edu.cn.
}

DOI: $10.4187 /$ respcare.07489 
People with COPD suffer from dyspnea, which also contributes to anxiety. Gift and Cahill ${ }^{4}$ provided evidence of a direct relationship between dyspnea and anxiety, suggesting that if one decreases, the other may decrease as well. Poor sleep quality is also one of the most common symptoms among individuals with COPD, leading to worse quality of life and more depression or anxiety. ${ }^{5}$

The Global Initiative for Chronic Obstructive Lung Disease guidelines state that pulmonary rehabilitation is a Class A method to improve dyspnea, health status, and exercise endurance in patients with COPD. ${ }^{6}$ However, the primary deterrent to effective aerobic training is dyspnea, an uncomfortable awareness of difficulty in breathing, which generally leads to a limitation of daily activities. ${ }^{7}$ Moreover, a qualitative study found that people with COPD declined pulmonary rehabilitation because it did not fit their perception of health, and they worried that participation may be time-consuming and conflict with daily activities. ${ }^{8}$ Thus, improving the adherence of patients with COPD to pulmonary rehabilitation, reducing symptoms, and promoting quality of life and functional ability are urgent problems to be solved.

Music therapy, which serves as an adjunct to medical care, can be used effectively for the treatment of lung conditions. It is enjoyable and low-cost and can be undertaken virtually anywhere. Music therapy involving breathing exercises is associated with decreased bronchial resistance and reduced anxiety levels in patients with asthma. ${ }^{9}$ It can improve adherence with intravenous infusions and reduce anxiety in children with severe pneumonia. ${ }^{10}$ Passive music therapy (ie, listening to music) is effective in relieving dyspnea and anxiety due to its relaxation effects, and thus promotes increased exercise tolerance in subjects with COPD ${ }^{11,12}$ Keeler et al $^{13}$ reported that music therapy consisting of singing can significantly increase oxytocin neuropeptide, a key hormone involved in human social and emotional processing. In another study, Kreutz et $\mathrm{al}^{14}$ reported that music therapy was associated with enhanced human immune function through the secretion of immunoglobulin A.

A systematic review of the research literature on music therapy in COPD was completed by Lee et $\mathrm{al}^{15}$ in 2015. However, there were some limitations, including a lack of Chinese data, as the findings from this review may be limited by the fact that studies were included only if they were published in English; a failure to analyze other symptoms, like dyspnea, anxiety, depression, quality of sleep, and physiological parameters (blood pressure and breathing frequency); and the availability of additional data for analysis. Therefore, we aimed to perform a systematic review and meta-analysis of previous studies to determine the effects of music therapy in adults with COPD.

\section{Methods}

All randomized controlled trials with full-text availability that compared music therapy with usual care or other non-musical types of intervention in subjects with COPD were included in the study. Inclusion criteria were medical diagnosis of COPD (ie, physician diagnosis and spirometry with $\mathrm{FEV}_{1} / \mathrm{FVC}<0.70$ ); stable clinical state, with no exacerbations; referred to music therapy in the form of distractive auditory stimuli (passive music) or combination of listening and singing (mixed music); and published in English or Chinese. Exclusion criteria were a predominant diagnosis other than COPD (eg, asthma, bronchiectasis, interstitial lung disease); an inability to hear music without assistive devices (eg, hearing aids); published as abstract only, or unpublished data; or a non-randomized controlled trial (eg, randomized crossover trial or cohort study).

We searched PubMed, Cochrane, Embase, Web of Science, China National Knowledge Infrastructure, VIP, and Wanfang Data from their inception to June 26, 2019. The search terms were as follows: ("music" OR "music therapy" OR "music*" OR "distractive stimuli" OR "auditory stimuli") AND ("COPD” OR "chronic obstructive pulmonary disease" OR "chronic air flow obstruction").

Two review authors ( $\mathrm{JH}$ and $\mathrm{XY}$ ) independently examined titles and abstracts retrieved from the search and selected all potentially eligible studies. Then the same review authors reviewed the full-text articles independently against the inclusion and exclusion criteria. A third review author (NZ) acted as an arbiter when consensus could not be reached. Two reviewers independently extracted the characteristics of the studies, including the author information, year of publication, study design, subjects, intervention, comparator, and outcomes.

The risk of bias was assessed independently by 2 reviewers using the Cochrane Handbook for Systematic Reviews of Interventions. ${ }^{16}$ We assessed the risk of bias according to the following domains: (1) random se-quence generation; (2) allocation concealment; (3) blinding of subjects and personnel; (4) blinding of outcome assessment; (5) incomplete outcome data; (6) selective outcome reporting; (7) other bias. The results of the assessment were judged as low risk, unclear risk, and high risk.

Statistical analysis was conducted using RevMan 5.3 (The Cochrane Collaborative, Copenhagen, Denmark). We primarily chose the changes that occurred between the beginning and the end of the intervention; if the baseline data were not available, we used the scores obtained at the end points. We used a chi-square test to estimate the heterogeneity of the mean difference (MD) or standardized mean difference (SMD) if data were reported using different units of measurement. A random-effect model was used to interpret the results if heterogeneity was statistically significant, whereas a fixed-effect model was used if heterogeneity 


\section{MUSIC THERAPY IN COPD}

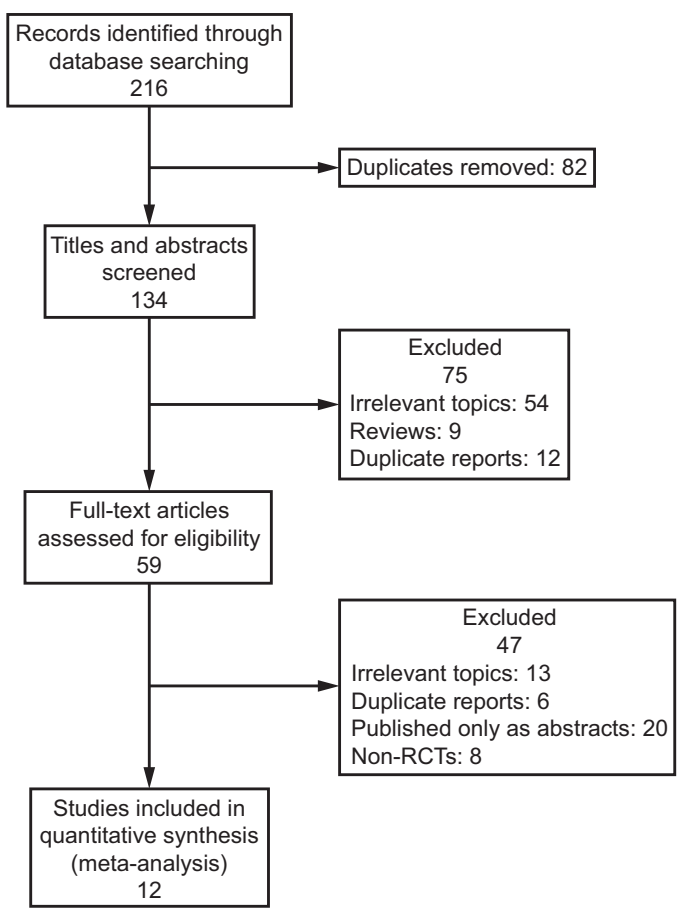

Fig. 1. Flow chart. RCT = randomized controlled trial.

was not statistically significant. For all results, $P<.05$ was considered to indicate a statistically significant difference. Subgroup analyses and sensitivity analysis were performed for achieving high-quality studies. Funnel plots were used to screen for potential publication bias.

\section{Results}

We retrieved 216 records through the database search. After removing duplicates and irrelevant references by screening titles and abstracts, 59 studies were assessed for eligibility. Forty-seven articles were excluded after full-text review, including irrelevant topics, records published only as abstracts, duplicate records, non-randomized controlled trials (eg, randomized crossover trials, a cohort study). Thus, 12 studies met the inclusion criteria and were included in the final meta-analysis (Fig. 1).

The characteristics of the included studies are outlined in Table 1, and the risk of bias of the included randomized controlled trials is summarized in Figure 2 and Figure 3. Among these studies, 2 studies explored the role of mixed music (combination of listening and singing) in treating COPD, ${ }^{20,22}$ while others were related to passive music. One study tested 2 music tempos, a slow tempo ( $60-89$ beats/min) or a moderate tempo (90-120 beats/min), compared to a control group during upper-extremity training. ${ }^{17}$ In another study, subjects were separated into 3 groups: a Turkish classical music group, a Western classical music group, and a control group. ${ }^{12}$ Three studies applied listening to music during walking-based training, ${ }^{11,18,21}$ while one used music during upper-extremity training. ${ }^{17}$ Most studies reported random allocation ${ }^{18,19,21,23,24,26}$ and similarities between groups at baseline, ${ }^{11,12,17-26}$ but only 1 study mentioned subject, therapist, or assessor blinding, ${ }^{23}$ due to the particularity of the music intervention (see the supplementary materials at http://www.rcjournal.com).

The modified Borg scale was used to evaluate dyspnea. Three studies ${ }^{11,18,21}$ provided numerical data for dyspnea and were included in the meta-analysis. The data indicated that there was low heterogeneity $\left(P=.49, I^{2}=0 \%\right)$; hence, a fixed-effects model was used. Pooled results showed a dyspnea reduction in the music group compared to the control group (MD: $-0.69,95 \% \mathrm{CI}-0.80$ to $-0.58 ; P<.001$ ) (Fig. 4).

Seven studies ${ }^{11,12,19,20,24-26}$ provided numerical data for anxiety scores and were included in the meta-analysis. When different scales were used for anxiety, we adopted the standardized mean difference (SMD) rather than the mean difference (MD). Analysis of the data indicated that there was heterogeneity $\left(P<.001 ; I^{2}=93 \%\right)$; hence, a random-effect model was used. Pooled results showed that there was a significant difference in anxiety between the 2 groups (SMD: $-1.87,95 \% \mathrm{CI}-2.72$ to $-1.02, P<.001$ ) (Fig. 5). In the analysis of depression, no significant difference was observed between music and controls (SMD: $0.96,95 \% \mathrm{CI}-2.14$ to $0.22, P=.11$ ) (see the supplementary materials at http://www.rcjournal.com).

In the analysis of quality of life, we identified 4 studies $^{11,17,21,23}$ that reported results that could be pooled for meta-analysis for the St George Respiratory Questionnaire. There was no statistically significant improvement in the St George Respiratory Questionnaire total score (MD: -2.58 , $95 \% \mathrm{CI}-12.65$ to $7.50, P=.62$ ), nor any change in symptoms, activity, or impact scores. However, in the analysis of the 3 studies ${ }^{11,18,25}$ that provided numerical data for quality of life and were included, there was improvement of this outcome (SMD: 3.31, 95\% CI 0.45 to $6.16, P=.02$ ) (see the supplementary materials at http://www.rcjournal.com).

Only 1 study $^{22}$ mentioned results for sleep quality. There was a statistically significant improvement in the Pittsburgh Sleep Quality Index total score $(P<.001)$, as well as subjective sleep quality, sleep latency, sleep duration, habitual sleep efficiency, and daytime dysfunction scores in the music group, while there was no significant improvement in sleep disturbance or use of sleeping medication compared with the control group $(P>.05)$.

Breathing frequency was measured in 5 studies. ${ }^{12,19,20,23,24}$ Analysis of the data indicated that there was heterogeneity $\left(P<.001, I^{2}=82 \%\right)$; hence, a random-effect model was used. The pooled results showed that there was improvement in breathing frequency (MD: $-1.98,95 \% \mathrm{CI}-3.42$ to $0.55, P=.007)$. Meanwhile, 3 studies $^{12,19,20}$ reported measures of systolic blood pressure. Analysis of the data indicated that there was low heterogeneity $(P=.73$, 


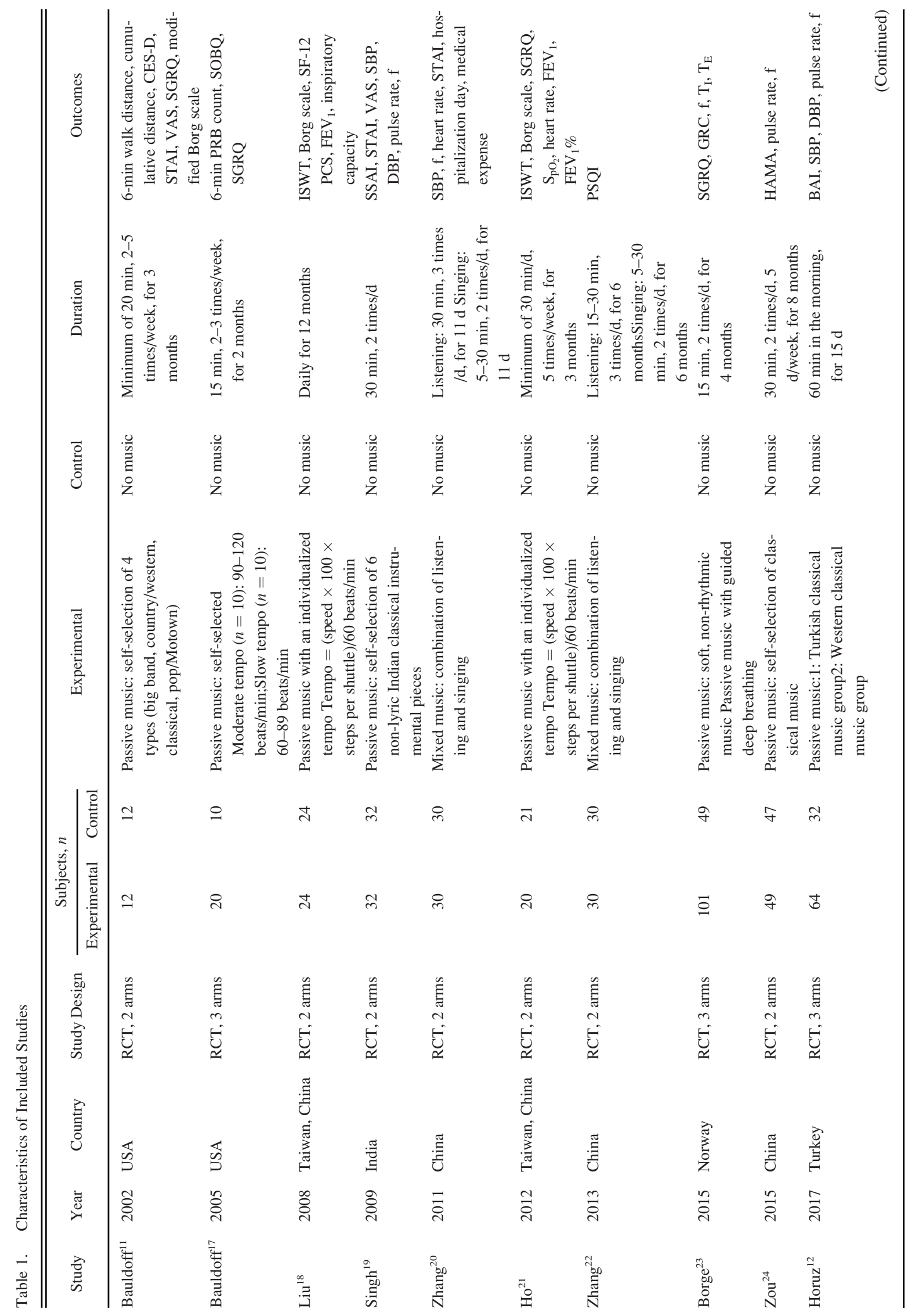


$I^{2}=0 \%$ ) using a fixed-effect model. The pooled results showed that there was significant improvement in systolic blood pressure (MD: $-7.45,95 \%$ CI -10.95 to 3.96; $P<.001)$. Two studies ${ }^{12,19}$ reported measures of diastolic pressure, and the pooled results showed that there was significant improvement in diastolic pressure in favor of the music group when compared to control (MD: $-4.07,95 \% \mathrm{CI}-7.03$ to $-1.12, P=.007$ ) (see the supplementary materials at http://www.rcjournal.com).

To determine the source of heterogeneity, a sensitivity analysis was performed by removing each study and redetermining the statistical significance of the results to assess whether any study substantially affected the results (see the supplementary materials at http://www.rcjournal. com). The results did not change significantly after removing each study in the analysis of anxiety, which indicated that the sensitivity was low and the results were stable and credible. We attributed the heterogeneity to including studies that used different scales. Similarly, the sensitivity was low in the analysis of breathing frequency. In the sensitivity analysis of St George Respiratory Questionnaire total score, after the exclusion of the study by Ho et al, ${ }^{21}$ the result was quite different (MD: 1.95, 95\% CI -5.83 to 9.72), which indicated that the sensitivity was higher. We believe the source of the high sensitivity was the incomplete outcome data because of a high loss to follow-up rate, with the case number of experimental and control data decreasing from 20 and 21 in the beginning to 15 and 15 in the end.

Two funnel plots with 10 studies were used to assess publication bias in the analysis. The results showed even distributions around the vertical, indicating no obvious publication bias (see the supplementary materials at http:// www.rcjournal.com).

\section{Discussion}

To our knowledge, this is the first meta-analysis to examine the effects of music therapy in the form of distractive auditory stimuli (ie, passive music) or a combination of listening and singing (ie, mixed music) on dyspnea, anxiety, depression, sleep quality, quality of life, and physiological parameters (ie, systolic and diastolic blood pressure, and breathing frequency) in adults with COPD, including Chinese subjects.

In total, 12 randomized controlled trials with 812 subjects with COPD were included in this review. Among them, 2 studies $^{20,22}$ referred to mixed music with a combination of listening and singing, while others were related to passive music. Sixty subjects with COPD in a study designed by Zhang et $\mathrm{al}^{20}$ were randomly assigned to a mixed music (combination of listening and singing) or a control group, and the results showed improvement in physiological outcomes, including systolic blood pressure, heart rate, and breathing frequency, as well as a reduction 


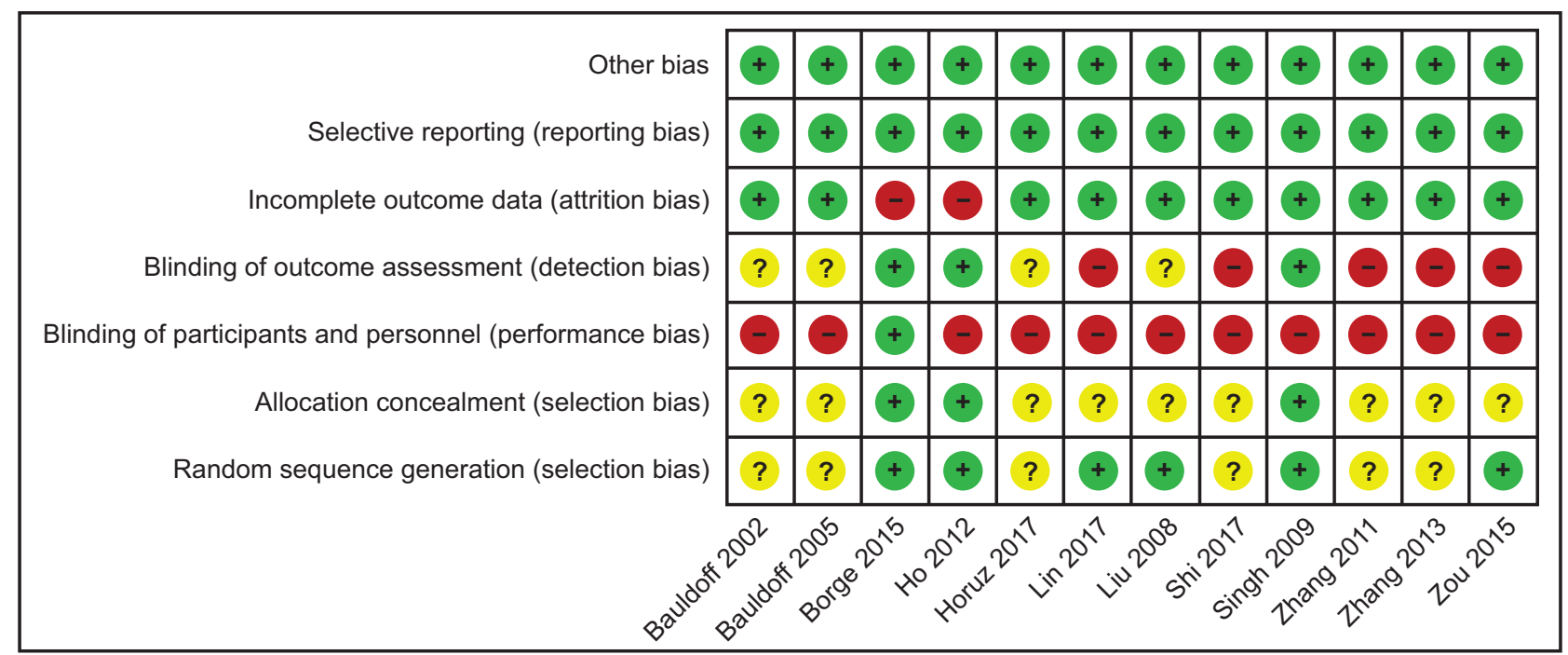

Fig. 2. Risk of bias summary.

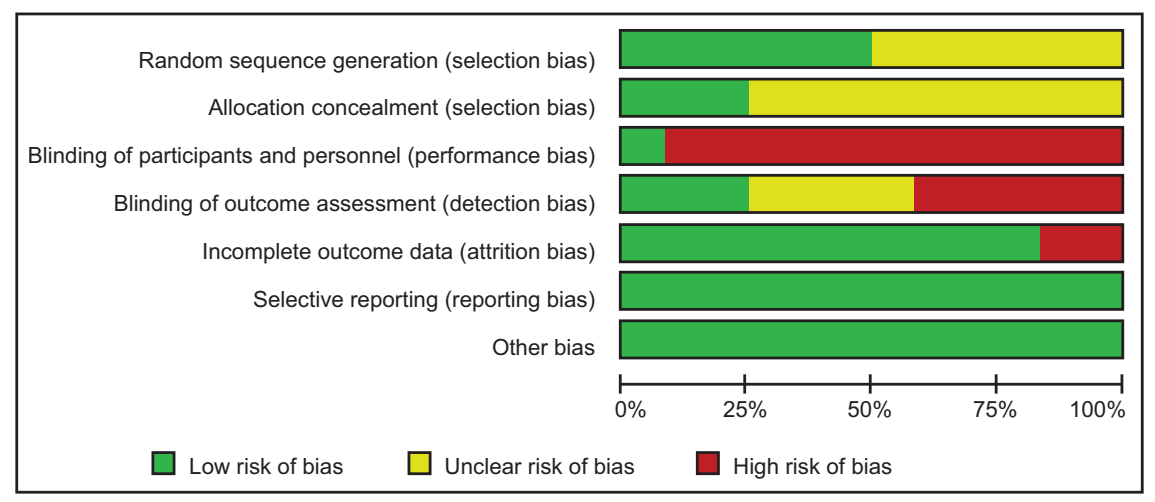

Fig. 3. Risk of bias.

in anxiety $(P<.05)$. These findings were consistent with passive music studies. ${ }^{12,20,24-26}$ In another study, Zhang et $\mathrm{al}^{22}$ investigated the influence of mixed music on sleep quality of subjects with stable COPD and reported statistically significant differences in the Pittsburgh Sleep Quality Index total score as well as subjective sleep quality, sleep latency, sleep duration, and habitual sleep efficiency score between 2 groups. This finding has not been replicated in passive music due to a lack of research in this field. In addition, 3 studies assessed pulmonary function in passive music therapy in subjects with COPD. In studies by Liu et $\mathrm{al}^{18}$ and Ho et al, ${ }^{21}$ there was no significant change in $\mathrm{FEV}_{1}$ in either group from baseline to the end of therapy. Meanwhile, the change in inspiratory capacity during exercise, representing dynamic hyperinflation, decreased significantly in the passive music group $(0.21 \pm 0.03 \mathrm{~L}, P<.001)$ compared with the control group $(0.33 \pm 0.02 \mathrm{~L})$ after 12 weeks of endurance exercise training at home. ${ }^{18}$
Contrary to these studies, Shi et $\mathrm{al}^{25}$ reported that music therapy had positive effects on $\mathrm{FEV}_{1}, \mathrm{P}_{\mathrm{aO}}$, and forced expiratory flow. These differences may result from methodological variations such as music genre and duration.

This meta-analysis showed for the first time that music therapy is effective in relieving dyspnea. Dyspnea, characterized by distress and unpleasantness, is the primary symptom that limits exercise tolerance in patients with COPD. ${ }^{27}$ Studies have indicated that the central processing area for dyspnea perception and auditory stimuli is partly shared. ${ }^{28-30}$ Music listening may reduce the sensation of dyspnea, thus promoting exercise capacity. Nineteen participants with COPD in a study designed by Lee et $\mathrm{al}^{31}$ completed 2 endurance walk tests, one with listening to self-selected music throughout the test and one without listening to music. The results showed that the mean $\pm \mathrm{SD}$ endurance time was longer (1.10 [95\% CI 0.41-1.78]) with listening to music ( $7.0 \pm$ $3.1 \mathrm{~min})$ than without $(5.9 \pm 2.6 \mathrm{~min})$, and the end-test 


\section{MUSIC THERAPY IN COPD}

\begin{tabular}{|c|c|c|c|c|c|c|c|c|c|c|c|}
\hline \multirow[b]{2}{*}{ Study or Subgroup } & \multicolumn{3}{|c|}{ Music } & \multicolumn{3}{|c|}{ Control } & \multicolumn{2}{|r|}{ Mean Difference } & \multirow{2}{*}{\multicolumn{2}{|c|}{$\begin{array}{c}\text { Mean Difference } \\
\text { IV, Fixed }(95 \% \mathrm{CI})\end{array}$}} & \\
\hline & Mean & SD & Total & Mean & SD & Total & Weight, \% & IV, Fixed $(95 \% \mathrm{CI})$ & & & \\
\hline Bauldoff 2002 & 3 & 1.2 & 12 & 3.2 & 0.8 & 12 & 1.9 & $-0.20(-1.02$ to 0.62$)$ & & & \\
\hline Ho 2012 & 0.8 & 1.1 & 20 & 1.5 & 1.6 & 21 & 1.8 & $-0.70(-1.54$ to 0.14$)$ & & & \\
\hline Liu 2008 & 3.2 & 0.2 & 24 & 3.9 & 0.2 & 24 & 96.4 & $-0.70(-0.81$ to -0.59$)$ & & & \\
\hline Total $(95 \% \mathrm{Cl})$ & & & 56 & & & 57 & 100 & $-0.69(-0.80$ to -0.58$)$ & & & \\
\hline \multicolumn{9}{|c|}{ Heterogeneity: $\mathrm{Chi}^{2}=1.42, \mathrm{df}=2(P=.49) ; \mathrm{I}^{2}=0 \%$} & & & \\
\hline \multicolumn{9}{|c|}{ Test for overall effect: $Z=12.19(P<.001)$} & $\begin{array}{ll}-1 & -0.5\end{array}$ & 0.5 & 1 \\
\hline
\end{tabular}

Fig. 4. Forest plot of music versus control - dyspnea.

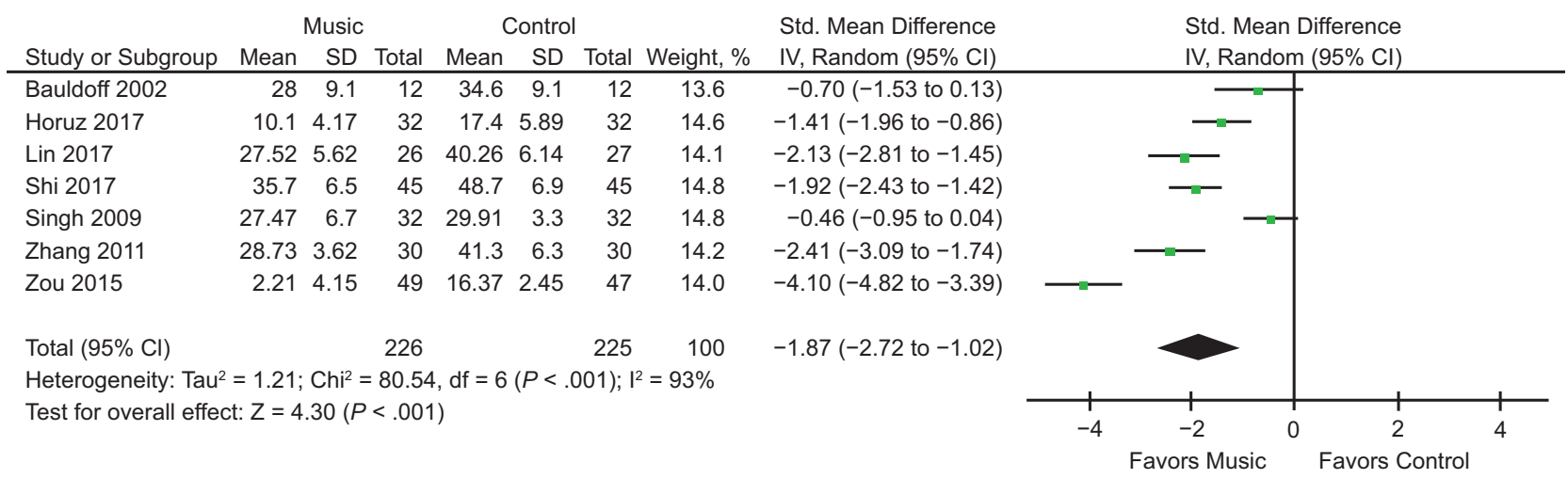

Fig. 5. Forest plot of music versus control - anxiety.

dyspnea (1.0 [95\% CI -2.80 to -1.80$])$ was also reduced. The study results of exercise capacity with passive music were similar to a previous systematic review by Lee et $\mathrm{al}^{15}$ in 2015. This research suggests a new non-pharmacologic approach to decrease dyspnea and improve exercise capacity in patients with COPD.

Pulmonary function was not measured in any mixedmusic studies (ie, a combination of listening and singing). To date, only 1 study (Bonilha et $\mathrm{al}^{32}$ ) has reported pulmonary function outcomes following singing training in subjects with COPD. The results showed no statistically significant differences between the singing group and control group for any of these measures, including $\mathrm{FEV}_{1} / \mathrm{FVC}$, end-respiratory volume, and inspiratory capacity. Meanwhile, there was a statistically significant improvement in peak expiratory pressure favoring the singing group. Singing has a positive effect on respiratory muscle training because it is dependent on the use of the lungs for air supply. ${ }^{33}$ However, further work is needed in subjects with COPD.

Some factors should be taken into account regarding this review, such as the different scales used in studies. Four studies whose subjects were assessed with the St George Respiratory Questionnaire reported no significant difference in quality of life between the music and control groups. ${ }^{11,17,21,23}$ These results were consistent with an earlier systematic review. ${ }^{15}$ However, 3 studies using Short Form-12, quality of life, or visual analog scale scores reached a different conclusion. ${ }^{11,18,25}$ According to the pooled analysis, music therapy was effective in improving total quality of life. These findings imply that different scales may influence results for efficacy of music therapy in adults with COPD. In addition, this was the main source of the high heterogeneity among those trials. Finally, no adverse reactions were reported in music intervention groups in any of the included studies, indicating that music therapy is safe for adults with COPD.

There are some limitations to our review. For example, in most of the included studies, music was chosen by subjects from a music library prepared by researchers without clear reason, so we do not have enough evidence to determine whether different types of music might lead to different results. In addition some studies reported a reduction in dyspnea with individualized, self-selected music ${ }^{7,34,35}$ rather than with investigator-selected music, ${ }^{36,37}$ which does not cater to individual preference. Self-selection of music tempo appears to maximize its beneficial effects. ${ }^{38}$ Additionally, the follow-up period of the included studies ranged from several days to 12 months; for most of the studies, this was too short a period to detect the long-term effects of music therapy in COPD. For this reason, large, high-quality clinical trials with longer follow-up periods are needed. 


\section{MUSIC THERAPY IN COPD}

\section{Conclusions}

Our meta-analysis suggests that music therapy can enhance potential therapeutic effects such as reducing dyspnea and relieving anxiety in subjects with COPD. Music therapy may be beneficial to sleep quality, including subjective sleep quality, sleep latency, sleep duration, and habitual sleep efficiency, although it makes no significant difference in St George Respiratory Questionnaire score. Compared with controls, music intervention did not lead to the relief of depression. Additionally, we found that music therapy is effective in lowering blood pressure and breathing frequency. Our results suggest that music therapy is a non-pharmacologic therapy that can be used with adults with COPD.

\section{REFERENCES}

1. GBD 2015 Chronic Respiratory Disease Collaborators. Global, regional, and national deaths, prevalence, disability-adjusted life years, and years lived with disability for chronic obstructive pulmonary disease and asthma - 1990-2015: a systematic analysis for the Global Burden of Disease Study 2015. Lancet Respir Med 2017;5(9):691706.

2. World Health Organization. Chronic obstructive pulmonary disease (COPD). Available at: http://www.who.int/respiratory/copd/burden/ en. Accessed July 8, 2018.

3. Wang C, Xu J, Yang L, Xu Y, Zhang X, Bai C, et al. Prevalence and risk factors of chronic obstructive pulmonary disease in China (the China Pulmonary Health $[\mathrm{CPH}]$ study) a national cross-sectional study. Lancet 2018;391(10131):1706-1717.

4. Gift AG, Cahill CA. Psychophysiologic aspects of dyspnea in chronic obstructive pulmonary disease: a pilot study. Heart Lung 1990;19 (3):252-257.

5. Chen R, Tian JW, Zhou LQ, Chen X, Yan HY, Zeng B, Zhang MS. The relationship between sleep quality and functional exercise capacity in COPD. Clin Respir J 2016;10(4):477-485.

6. Spruit MA, Singh SJ, Garvey C, ZuWallack R, Nici L, Rochester C, et al. An official American Thoracic Society/European Respiratory Society statement: key concepts and advances in pulmonary rehabilitation. Am J Respir Crit Care Med 2013;188(8):e13-e64.

7. Shingai K, Kanezaki M. Effect of dyspnea induced by breath-holding on maximal muscular strength of patients with COPD. J Phys Ther Sci 2014;26(2):255-258.

8. Mathar H, Fastholm P, Lange P, Larsen NS. Why do patients decline participation in offered pulmonary rehabilitation? A qualitative study. Clin Rehabil 2017;31(12):1674-1683.

9. Janiszewski M, Kronenberger M, Drozd B. Studies on the use of music therapy as a form of breathing exercise in bronchial asthma. Pol Merkuriusz Lek 1996;1(1):32-33.

10. Hou SL, Xing M. Effect of music therapy on negative emotion and treatment compliance in children with severe pneumonia. China Naturopathy 2019;27(10):24-26.

11. Bauldoff GS, Hoffman LA, Zullo TG, Sciurba FC. Exercise maintenance following pulmonary rehabilitation: effect of distractive stimuli. Chest 2002;122(3):948-954.

12. Horuz D, Kurcer MA, Erdoğan Z. The effect of music therapy on anxiety and various physical findings in patients with COPD in a pulmonology service. Holistic Nurs Pract 2017;31(6):378-383.

13. Keeler JR, Roth EA, Neuser BL, Spitsbergen JM, Waters DJM, Vinney JM. The neurochemistry and social flow of singing: bonding and oxytocin. Front Hum Neurosci 2015;9:518-527.
14. Kreutz G, Bongard S, Rohrmann S, Hodapp V, Grebe D. Effects of choir singing or listening on secretory immunoglobulin A, cortisol, and emotional state. J Behav Med 2004;27(6):623-635.

15. Lee AL, Desveaux L, Goldstein RS, Brooks D. Distractive auditory stimuli in the form of music in individuals with COPD: a systematic review. Chest 2015;148(2):417-429.

16. The Cochrane Collaboration; Higgins JPT, Green S, eds. The Cochrane Handbook for Systematic Reviews of Interventions, version 5.1. Available at: http://www.handbook-5-1.cochrane.org. Accessed October 13, 2018

17. Bauldoff GS, Rittinger M, Nelson T, Doehrel J, Diaz PT. Feasibility of distractive auditory stimuli on upper extremity training in persons with chronic obstructive pulmonary disease. J Cardiopulm Rehabil 2005;25(1):50-55.

18. Liu WT, Wang CH, Lin HC, Lin SM, Lee KY, Lo YL, et al. Efficacy of a cell phone-based exercise programme for COPD. Eur Respir J 2008;32(3):651-659.

19. Singh VP, Rao V, Prem V, Sahoo RC, Keshav KP. Comparison of the effectiveness of music and progressive muscle relaxation for anxiety in COPD: a randomized controlled pilot study. Chron Respir Dis 2009;6(4):209-216

20. Zhang YG, Peng XF, Shao H, Zhang SS, Liu YQ, Wang FY. Study on the role of adjuvant therapy of individual musicotherapy combined with listening and singing on patients with chronic obstructive pulmonary disease. Today Nurs 2011;12(9):3-4.

21. Ho CF, Maa SH, Shyu YIL, Lai YT, Hung TC, Chen HC. Effectiveness of paced walking to music at home for patients with COPD. COPD 2012;9(5):447-457.

22. Zhang YG, Shao H. Influence of music therapy on sleep quality of patients with stable chronic obstructive pulmonary disease. Chin Nurs Res 2013;27(22):2354-2355.

23. Borge CR, Mengshoel AM, Omenaas E, Moum T, Ekman I, Lein MP, et al. Effects of guided deep breathing on breathlessness and the breathing pattern in chronic obstructive pulmonary disease: a doubleblind randomized control study. Patient Educ Couns 2015;98(2):182190.

24. Zou X. Effect of music combined with drug therapy on anxiety symptoms in patients with chronic obstructive pulmonary disease. Chin J Phys Med Rehabil 2015;37(10):774-776.

25. Shi CC, Chen Y, Li XM. Observation on the effect of music intervention nursing therapy on patients with chronic obstructive pulmonary disease. Chin Community Doct 2017;33(18):136-137.

26. Lin L, Li S, Chen X, Qiu YZ. Effect of music on anxiety and dyspnea in patients with stable chronic obstructive pulmonary disease. Yunnan J Tradit Chin Med Mater Med 2017;38(8):104-105.

27. Parshall MB, Schwartzstein RM, Adams L, Banzett RB, Manning HL, Bourbeau J, et al. An official American Thoracic Society statement: update on the mechanisms, assessment, and management of dyspnea. Am J Respir Crit Care Med 2012;185(4):435-452.

28. Davenport PW, Vovk A. Cortical and subcortical central neural pathways in respiratory sensations. Respir Physiol Neurobiol 2009;167 (1):72-86.

29. von Leupoldt A, Dahme B. Cortical substrates for the perception of dyspnea. Chest 2005;128(1):345-354.

30. von Leupoldt A, Sommer T, Kegat S, Baumann HJ, Klose H, Dahme $\mathrm{B}$, Büchel C. The unpleasantness of perceived dyspnea is processed in the anterior insula and amygdala. Am J Respir Crit Care Med 2008;177(9):1026-1032.

31. Lee AL, Dolmage TE, Rhim M, Goldstein RS, Brooks D. The impact of listening to music during a high-intensity exercise endurance test in people with COPD. Chest 2018;153(5):1134-1141.

32. Bonilha AG, Onofre F, Vieira ML, Prado MY, Martinez JA. Effects of singing classes on pulmonary function and quality of life of COPD patients. Int J Chron Obstruct Pulmon Dis 2009;4:1-8. 


\section{MUSIC THERAPY IN COPD}

33. Lord VM, Cave P, Hume VJ, Flude EJ, Evans A, Kelly JL, et al. Singing teaching as a therapy for chronic respiratory disease-a randomised controlled trial and qualitative evaluation. BMC Pulm Med 2010;10:41.

34. von Leupoldt A, Taube K, Schubert-Heukeshoven S, Magnussen H, Dahme B. Distractive auditory stimuli reduce the unpleasantness of dyspnea during exercise patients with COPD. Chest 2007;132 (5):1506-1512.

35. Thornby MA, Haas F, Axen K. Effect of distractive auditory stimuli on exercise tolerance in patients with COPD. Chest 1995;107(5):1213-1217.
36. Reychler G, Mottart F, Boland M, Wasterlain E, Pieters T, Caty G, et al. Influence of ambient music on perceived exertion during a pulmonary rehabilitation session: a randomized crossover study. Respir Care 2015;60(5):711-717.

37. Kopacz M. Personality and music preferences: the influence of personality traits on preferences regarding musical elements. J Music Ther 2005;42(3):216-239.

38. Karageorghis CI, Priest DL. Music in the exercise domain: a review and synthesis (Part I). Int Rev Sport Exerc Psychol 2012; $5(1): 44-66$ 